\title{
STABILITY OF CYLINDRICAL PANEL WITH VARIABLE THICKNESS
}

\author{
Nguyen Thi Hien Luong and Thach Som So Hoach \\ Department of Civil Engineering, Ho Chi Minh University of Technology
}

\begin{abstract}
A linear buckling analysis based on the small deflection theory is presented for the cylindrical panel with sinusoidal changes in the shell thickness. The buckling load for simply supported cylindrical panel around the periphery is defined by using the hybrid perturbation - Galerkin method. The influence of the thickness variation parameter on the critical loads is investigated.
\end{abstract}

\section{INTRODUCTION}

In recent years, the study on stability of thin structural components with variable thickness has attracted attention to many researchers [1-6]. Elishakoff et al [2] studied the effect of axisymmetric imperfections in the shape of the axisymmetric buckling mode on the buckling of cylindrical shells. Ye Zhiming [3] introduced the nonlinear analysis and optimization of shallow shell of variable thickness. Yeh et al [4] treated chaotic and bifurcation dynamics for a simply supported rectangular plate of thermo-mechanical coupling in large deflection. Mateus et al [5] studied post-buckling behavior of corroded steel plates. Nguyen and Tran [6] investigated the stability of thin rectangular plates with variable thickness on a basis of the theory of thin plates of small deflections.

The major objective of this paper is to investigate in detail the buckling of cylindrical panel of variable thickness, based on the small deflection theory and shallow shells theory. The Hybrid Pertubation-Galerkin method is in use to determine the critical load factor of cylindrical panel with variable thickness. The influence of the thickness non-uniformity parameter to the buckling load is investigated. General asymptotic formulae for the buckling load are derived and numerical results are investigated for compressive simply supported panels.

\section{GOVERNING DIFFERENTIAL EQUATIONS}

Consider a cylindrical panel with small thickness variation loaded in its middle surface by uniform compression $N$ (Fig. 1). As the panel thickness is not uniform in the $x$ direction and radius of cylindrical panel is $R$, the governing differential equations with variable coefficients for the panels in general case are obtained as follows:

Assume that: $N_{i j}^{0}, \varepsilon_{i j}^{0}, W^{0}(i, j=1,2)$ are stretching forces, strains in the middle surface, and the radial deflection in the fundamental pre-buckling state; $N_{i j}^{1}, M_{i j}^{1}, \varepsilon_{i j}^{1}$, $W^{1}(i, j=1,2)$ are stretching forces, moments, strains in the middle surface, the radial deflection in the adjacent buckling state. We have the increments of solutions at buckling:

$$
\begin{aligned}
& N_{i j}=N_{i j}^{1}-N_{i j}^{0}, M_{i j}=M_{i j}^{1}-M_{i j}^{0}=M_{i j}^{1}, \\
& \varepsilon_{i j}=\varepsilon_{i j}^{1}-\varepsilon_{i j}^{0}, W=W^{1}-W^{0}=W^{1}, \quad(i, j=1,2) .
\end{aligned}
$$


It is noted that in the buckling state: $M_{i j}^{0}=0, W^{0}=0$.

We shall use the basic general equations in term of increments to solve the stability problem of cylindrical panel compressed in the direction of long edges.

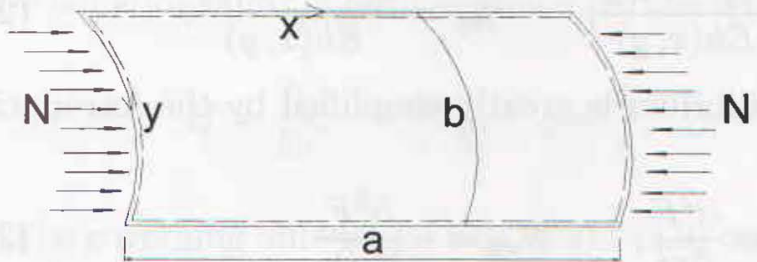

Fig. 1. Uniaxially compressed cylindrical panel

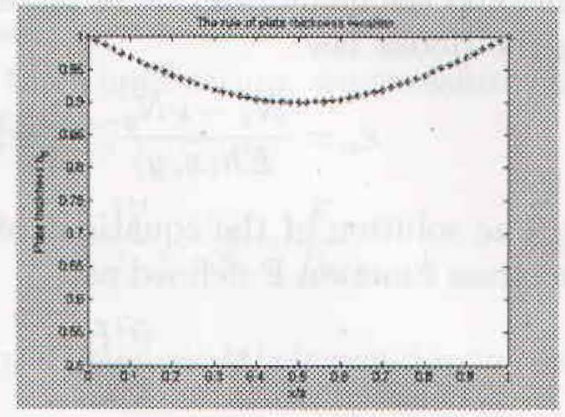

Fig. 2. Expression graph of thickness variation $h(x)$ when $\varepsilon=0.1$

In the case of small deflection, the strain - displacement increments relations are of the forms [1], [2]:

$$
\varepsilon_{x}=\frac{\partial U}{\partial x}, \quad \varepsilon_{y}=\frac{\partial V}{\partial y}-\frac{W}{R}, \quad \gamma_{x y}=\frac{\partial U}{\partial y}+\frac{\partial V}{\partial x}
$$

where $U, V$ are displacements in $x$ and $y$ directions.

By taking the second derivative of these expressions and combining the resulting expressions, we obtain the compatibility equation:

$$
\frac{\partial^{2} \varepsilon_{x}}{\partial y^{2}}-\frac{\partial^{2} \gamma_{x y}}{\partial x \partial y}+\frac{\partial^{2} \varepsilon_{y}}{\partial x^{2}}=-\frac{1}{R} \frac{\partial^{2} W}{\partial x^{2}}
$$

We use the stability equation in terms of bending and twisting moment increments [7] in the case of cylindrical panel:

$$
\frac{\partial^{2} M_{x}}{\partial x^{2}}-2 \frac{\partial^{2} M_{x y}}{\partial x \partial y}+\frac{\partial^{2} M_{y}}{\partial y^{2}}=-\left[N_{x} \frac{\partial^{2} W}{\partial x^{2}}-N_{x}^{0} \frac{\partial^{2} W}{\partial x^{2}}+N_{y}\left(\frac{\partial^{2} W}{\partial y^{2}}+\frac{1}{R}\right)+2 N_{x y} \frac{\partial^{2} W}{\partial x \partial y}\right] .
$$

In deriving the buckling equation of cylindrical panel of variable thickness, we assume that there is no abrupt variation in thickness so that the expressions for bending and twisting moments increments derived for panels of constant thickness apply with sufficient accuracy to this case also. Then:

$$
\begin{aligned}
& M_{x}=-D(x, y)\left(\frac{\partial^{2} W}{\partial x^{2}}+\nu \frac{\partial^{2} W}{\partial y^{2}}\right) \\
& M_{y}=-D(x, y)\left(\frac{\partial^{2} W}{\partial y^{2}}+\nu \frac{\partial^{2} W}{\partial x^{2}}\right) \\
& M_{x y}=D(x, y)(1-\nu) \frac{\partial^{2} W}{\partial x \partial y}
\end{aligned}
$$


where $D(x, y)=\frac{E h^{3}(x, y)}{12\left(1-\nu^{2}\right)}$.

We express the relations of strain components in the middle surface of the cylindrical panel and the membrane forces increments $N_{x}, N_{y}, N_{x y}$ by the known formulas representing the Hooke law:

$$
\varepsilon_{x}=\frac{N_{x}-\nu N_{y}}{E h(x, y)}, \quad \varepsilon_{y}=\frac{N_{y}-\nu N_{x}}{E h(x, y)}, \quad \gamma_{x y}=\frac{2(1+\nu) N_{x y}}{E h(x, y)} .
$$

The solution of the equations of equilibrium is greatly simplified by the introducing the stress function $\mathrm{F}$ defined as:

$$
N_{x}=\frac{\partial^{2} F}{\partial y^{2}}, \quad N_{y}=\frac{\partial^{2} F}{\partial x^{2}}, \quad N_{x y}=-\frac{\partial^{2} F}{\partial x \partial y} .
$$

Substituting Eq.(2.6) into Eq.(2.3) and taking into account Eq.(2.7), we obtain:

$$
\begin{aligned}
& h^{2} \nabla^{2} \nabla^{2} F-2 h \frac{\partial h}{\partial x}\left(\frac{\partial^{3} F}{\partial x^{3}}+\frac{\partial^{3} F}{\partial x \partial y^{2}}\right)+\left[2\left(\frac{\partial h}{\partial x}\right)^{2}-h \frac{\partial^{2} h}{\partial x^{2}}\right]\left(\frac{\partial^{2} F}{\partial x^{2}}-\nu \frac{\partial^{2} F}{\partial y^{2}}\right)- \\
& -2 h \frac{\partial h}{\partial y}\left(\frac{\partial^{3} F}{\partial y^{3}}+\frac{\partial^{3} F}{\partial x^{2} \partial y}\right)+\left[2\left(\frac{\partial h}{\partial y}\right)^{2}-h \frac{\partial^{2} h}{\partial y^{2}}\right]\left(\frac{\partial^{2} F}{\partial y^{2}}-\nu \frac{\partial^{2} F}{\partial x^{2}}\right)+ \\
& +4(1+\nu) \frac{\partial h}{\partial x} \frac{\partial h}{\partial y} \frac{\partial^{2} F}{\partial x \partial y}-2(1+\nu) h \frac{\partial^{2} h}{\partial x \partial y} \frac{\partial^{2} F}{\partial x \partial y}=E h^{3}\left(-\frac{1}{R} \frac{\partial^{2} W}{\partial x^{2}}\right) .
\end{aligned}
$$

When the cylindrical panel is under the action of uniform axial compression (Fig. 1), substituting Eq. (2.5) in the buckling Eq. (2.4) and observing that the flexural rigidity $D$ is no longer a constant but a function of coordinate $x$, we obtain:

$$
\begin{aligned}
& \frac{E h^{3}}{12\left(1-\nu^{2}\right)} \nabla^{2} \nabla^{2} W+\frac{6 E h^{2}}{12\left(1-\nu^{2}\right)} \frac{\partial h}{\partial x}\left(\frac{\partial^{3} W}{\partial x^{3}}+\frac{\partial^{3} W}{\partial x \partial y^{2}}\right)+\frac{3 E h^{2}}{12\left(1-\nu^{2}\right)} \frac{\partial^{2} h}{\partial x^{2}}\left(\frac{\partial^{2} W}{\partial x^{2}}+\nu \frac{\partial^{2} W}{\partial y^{2}}\right)+ \\
& +\frac{6 E h}{12\left(1-\nu^{2}\right)}\left(\frac{\partial h}{\partial x}\right)^{2}\left(\frac{\partial^{2} W}{\partial x^{2}}+\nu \frac{\partial^{2} W}{\partial y^{2}}\right)+\frac{6 E h}{12\left(1-\nu^{2}\right)}\left(\frac{\partial h}{\partial y}\right)^{2}\left(\frac{\partial^{2} W}{\partial y^{2}}+\nu \frac{\partial^{2} W}{\partial x^{2}}\right)+ \\
& +\frac{6 E h^{2}}{12\left(1-\nu^{2}\right)} \frac{\partial h}{\partial y}\left(\frac{\partial^{3} W}{\partial y^{3}}+\frac{\partial^{3} W}{\partial x^{2} \partial y}\right)+\frac{3 E h^{2}}{12\left(1-\nu^{2}\right)} \frac{\partial^{2} h}{\partial y^{2}}\left(\frac{\partial^{2} W}{\partial y^{2}}+\nu \frac{\partial^{2} W}{\partial x^{2}}\right)+ \\
& +\frac{E h^{2}}{2(1+\nu)} \frac{\partial^{2} h}{\partial x \partial y} \frac{\partial^{2} W}{\partial x \partial y}+\frac{E h}{(1+\nu)} \frac{\partial h}{\partial x} \frac{\partial h}{\partial y} \frac{\partial^{2} W}{\partial x \partial y}=-N_{x}^{0} \frac{\partial^{2} W}{\partial x^{2}}+\frac{1}{R} \frac{\partial^{2} F}{\partial x^{2}}
\end{aligned}
$$

with $\nabla=\frac{\partial^{2}}{\partial x^{2}}+\frac{\partial^{2}}{\partial y^{2}}$.

In Eq. (2.9) $W$ and $F$ represent the displacement and the stress function, $\nu$ is Poisson's ratio, $E$ is the modulus of elasticity, $R$ is the radius of cylindrical panel. Eqs.(2.8)-(2.9) constitute the governing differential equations for small deflections of cylindrical panel with variable thickness.

In Eqs.(2.8)-(2.9), $h$ is the cylindrical panel thickness, which is assumed here varying with sine function in $x$ direction :

$$
h(x)=h_{0}\left(1-\varepsilon \sin \frac{p \pi x}{a}\right) \quad \text { with } \varepsilon \geq 0
$$


where $h_{0}$ is the cylindrical panel thickness and $\varepsilon, p$ are the non-dimensional parameters indicating the magnitude and wave of the thickness variation, respectively. When $x=0$ and $x=a$, one has $h(x)=h_{0}$, for the case $x=a / 2$ : one has $h(x)=h_{0}(1-\varepsilon)$ (Fig. 2). The thickness parameter $\varepsilon$ varies from zero to 0.2 and is positive in order to achieve a detrimental effect by a "thinning" of the wall thickness.

To make the resulting solutions more general in their application, we transform the above equations into non-dimensional parameters as follows:

$$
H=\frac{h}{h_{0}}, \quad r=\frac{a}{b}, \quad \xi=\frac{x}{a}, \quad \eta=\frac{y}{b}, \quad w=\frac{W}{h_{0}}, \quad f=\frac{F}{D_{0}} .
$$

The governing differential Eqs.(2.8) - (2.9) can be rewritten into their non-dimensional form:

$$
\begin{aligned}
& H^{2}\left(\frac{\partial^{4} f}{\partial \xi^{4}}+2 r^{2} \frac{\partial^{4} f}{\partial \xi^{2} \partial \eta^{2}}+r^{4} \frac{\partial^{4} f}{\partial \eta^{4}}\right)-2 H \frac{d H}{d \xi}\left(\frac{\partial^{3} f}{\partial \xi^{3}}+r^{2} \frac{\partial^{3} f}{\partial \xi \partial \eta^{2}}\right)+ \\
& +2\left(\frac{d H}{d \xi}\right)^{2}\left(\frac{\partial^{2} f}{\partial \xi^{2}}-\nu r^{2} \frac{\partial^{2} f}{\partial \eta^{2}}\right)-H \frac{d^{2} H}{d \xi^{2}}\left(\frac{\partial^{2} f}{\partial \xi^{2}}-\nu r^{2} \frac{\partial^{2} f}{\partial \eta^{2}}\right)=-\frac{12\left(1-\nu^{2}\right) H^{3} b^{2} r^{2}}{R h_{0}} \frac{\partial^{2} w}{\partial \xi^{2}} \\
& H^{3}\left(\frac{\partial^{4} w}{\partial \xi^{4}}+2 r^{2} \frac{\partial^{4} w}{\partial \xi^{2} \partial \eta^{2}}+r^{4} \frac{\partial^{4} w}{\partial \eta^{4}}\right)+6 H^{2} \frac{d H}{d \xi}\left(\frac{\partial^{3} w}{\partial \xi^{3}}+r^{2} \frac{\partial^{3} w}{\partial \xi \partial \eta^{2}}\right)+ \\
& +6 H\left(\frac{d H}{d \xi}\right)^{2}\left(\frac{\partial^{2} w}{\partial \xi^{2}}+\nu r^{2} \frac{\partial^{2} w}{\partial \eta^{2}}\right)+3 H^{2} \frac{d^{2} H}{d \xi^{2}}\left(\frac{\partial^{2} w}{\partial \xi^{2}}+\nu r^{2} \frac{\partial^{2} w}{\partial \eta^{2}}\right)= \\
& =-\frac{12\left(1-\nu^{2}\right) r^{2}}{E h_{0}^{3}}\left(b^{2} N_{x}^{0} \frac{\partial^{2} w}{\partial \xi^{2}}\right)+r^{2}\left(\frac{b^{2}}{R h_{0}} \frac{\partial^{2} f}{\partial \xi^{2}}\right)
\end{aligned}
$$

If the cylindrical panel is simply supported around the periphery, then the boundary conditions are:

Out-of plane conditions:

$$
\begin{aligned}
w & =0 \quad \text { at } \xi=0 ; 1 \quad \text { and } \eta=0 ; 1, \\
M_{\xi} & =-D(\xi)\left[\frac{\partial^{2} w}{a^{2} \partial \xi^{2}}+\nu \frac{\partial^{2} w}{b^{2} \partial \eta^{2}}\right]=0 \text { at } \quad \xi=0 ; \mathbf{1}, \\
M_{\eta} & =-D(\xi)\left[\frac{\partial^{2} w}{b^{2} \partial \eta^{2}}+\nu \frac{\partial^{2} w}{a^{2} \partial \xi^{2}}\right]=0 \text { at } \quad \boldsymbol{\eta}=\mathbf{0} ; \mathbf{1},
\end{aligned}
$$

with $D(\xi)=\frac{E h^{3}}{12\left(1-\nu^{2}\right)}=\frac{E\left[h_{0}(1-\varepsilon \sin (p \pi \xi)]^{3}\right.}{12\left(1-\nu^{2}\right)}$.

In -plane boundary displacement conditions [1] have the form:

$$
v(\xi, \eta)=\int\left[\frac{D_{0} b}{E h}\left(\frac{\partial^{2} f}{a^{2} \partial \xi^{2}}-\nu \frac{\partial^{2} f}{b^{2} \partial \eta^{2}}\right)+\frac{w h_{0} b}{R}\right] d \eta=0 \quad \text { at } \quad \xi=0 ; 1
$$


In -plane boundary stress conditions:

$$
\begin{aligned}
& \frac{\partial^{2} f}{b^{2} \partial \eta^{2}}=0 \quad \text { at } \xi=0 ; 1 \\
& \frac{\partial^{2} f}{a b \partial \xi \partial \eta}=0 \quad \text { at } \quad \xi=0 ; 1 \text { and } \eta=0 ; 1 .
\end{aligned}
$$

\section{HYBRID PERTUBATION - GALERKIN METHOD}

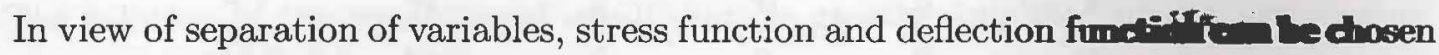
satisfying the boundary conditions $(2.14 \mathrm{a}),(2.14 \mathrm{~b})$ and $(2.14 \mathrm{c})$ as :

$$
\begin{aligned}
f(\xi, \eta) & =\bar{f}(\xi) \sin (n \pi \eta), \\
w(\xi, \eta) & =\bar{w}(\xi) \sin (n \pi \eta),
\end{aligned}
$$

with $\bar{w}(\xi)=\sum_{m=1}^{M} A_{m} \sin (m \pi \xi)$ and $m, n$ denotes the numbers of wase $y$ direction; $m, n=1$ are integers (in this paper: $m=n=1$ ).

The boundary conditions (2.16b) are satisfied in the form:

$$
\int_{0}^{1} \frac{\partial^{2} f(\xi, \eta)}{\partial \xi \partial \eta} d \eta=0, \quad \int_{0}^{1} \frac{\partial^{2} f(\xi, \eta)}{\partial \xi \partial \eta} d \xi=\mathbf{0} .
$$

The boundary conditions (2.15) and (2.16a) can be rewriten into the

$$
\frac{\partial^{2} \bar{f}(\xi)}{\partial \xi^{2}}=0 \text { and } \bar{f}(\xi)=0 \quad \text { at } \quad \xi=0 ; 1
$$

Equations (2.12) and (2.13) are thus transformed into ordinary differential eq tions:

$$
\begin{aligned}
& H^{2} \bar{f}^{4}-2 H \frac{d H}{d \xi} \bar{f}^{\prime \prime \prime}+\left[2\left(\frac{d H}{d \xi}\right)^{2}-2 r^{2} N^{2} H^{2}-H \frac{d^{2} H}{d \xi^{2}}\right] \bar{f}^{\prime \prime}+2 r^{2} \boldsymbol{N}^{2} \boldsymbol{H} \frac{\boldsymbol{d H}}{\boldsymbol{d \xi}} \boldsymbol{f}^{\prime}+ \\
& +\left[r^{4} N^{4} H^{2}+2 \nu r^{2} N^{2}\left(\frac{d H}{d \xi}\right)^{2}-\nu r^{2} N^{2} H \frac{d^{2} H}{d \xi^{2}}\right] \bar{f}=-\frac{12\left(1-\nu^{2}\right) \boldsymbol{H}^{3} r^{2} b^{2}}{R h_{0}}, \\
& H^{3} \bar{w}^{4}+6 H^{2} \frac{d H}{d \xi} \bar{w}^{\prime \prime \prime}+\left[-6 r^{2} N^{2} H^{2} \frac{d H}{d \xi}\right] \vec{w}^{\prime}+ \\
& +\left[6 H\left(\frac{d H}{d \xi}\right)^{2}+3 H^{2} \frac{d^{2} H}{d \xi^{2}}-2 r^{2} N^{2} H^{3}+\frac{r^{2} b^{2} N_{x}^{0}}{D_{0}}\right] \bar{w}^{\prime \prime}+ \\
& +\left[r^{4} N^{4} H^{3}-6 \nu r^{2} N^{2} H\left(\frac{d H}{d \xi}\right)^{2}-3 \nu r^{2} N^{2} H^{2} \frac{d^{2} H}{d \xi^{2}}\right] \bar{w}-\frac{\boldsymbol{b}^{2}}{R \boldsymbol{h}_{\mathbf{0}}} \widehat{\boldsymbol{f}}^{\prime \prime}=\mathbf{0},
\end{aligned}
$$

with $N=n \pi$. 
In order to solve the compatibility equation (3.4) for $\bar{f}$, the pertubation procedure will be employed here. To this end, $\bar{f}$ is expressed in term of the thickness variation parameter $\varepsilon$ as:

$$
\bar{f}(\xi)=f_{0}(\xi)+\varepsilon f_{1}(\xi) .
$$

Substituting (3.6) into (3.4) and keeping (2.10) in mind, after collecting the like terms $\varepsilon$ :

$$
\begin{aligned}
& f_{0}^{4}-2 N^{2} r^{2} f_{0}^{\prime \prime}+N^{4} r^{4} f_{0}+12\left(1-\nu^{2}\right) \bar{w}^{\prime \prime} r^{2} \frac{b^{2}}{R h_{0}}+\varepsilon\left[f_{1}^{4}-2 N^{2} r^{2} f_{1}^{\prime \prime}+N^{4} r^{4} f_{1}-\right. \\
& -P^{2} f_{0}^{\prime \prime} \sin (N \xi)+2 P f_{0}^{\prime \prime \prime} \cos (P \xi)-36\left(1-\nu^{2}\right) r^{2} \frac{b^{2}}{R h_{0}} \bar{w}^{\prime \prime} \sin (P \xi)-2 f_{0}^{4} \sin (N \xi)+ \\
& \left.+4 N^{2} r^{2} f_{0}^{\prime \prime} \sin (P \xi)-2 N^{2} r^{2} f_{0}^{\prime} P \cos (N \xi)-P^{2} N^{2} \nu r^{2} f_{0} \sin (P \xi)-2 N^{4} r^{4} f_{0} \sin (P \xi)\right]=0,
\end{aligned}
$$

with $P=p \pi$.

From equation (3.7), we obtain:

$$
\begin{aligned}
& L\left(f_{0}\right)=-12\left(1-\nu^{2}\right) \bar{w}^{\prime \prime} r^{2} \frac{b^{2}}{R h_{0}} \\
& L\left(f_{1}\right)=P^{2} f_{0}^{\prime \prime} \sin (N \xi)-2 P f_{0}^{\prime \prime \prime} \cos (P \xi)+36\left(1-\nu^{2}\right) r^{2} \frac{b^{2}}{R h_{0}} \bar{w}^{\prime \prime} \sin (P \xi)+2 f_{0}^{4} \sin (N \xi)- \\
& -4 N^{2} r^{2} f_{0}^{\prime \prime} \sin (P \xi)+2 N^{2} r^{2} f_{0}^{\prime} P \cos (N \xi)+P^{2} N^{2} \nu r^{2} f_{0} \sin (P \xi)+2 N^{4} r^{4} f_{0} \sin (P \xi)
\end{aligned}
$$

where the operator $L(\bullet)$ is defined as:

$$
L(f)=f^{4}-2 N^{2} r^{2} f^{\prime \prime}+N^{4} r^{4} f .
$$

Equations (3.8) - (3.10) and the boundary condition (3.3) can be solved analytically with the aid of the computerized symbolic algebra Matlab for $f_{0}$ and $f_{1}$ :

In case: $m=n=p=1$, we have:

$$
f_{0}=\frac{a_{10}}{\pi^{4}\left(r^{2}+1\right)^{2}} \sin (\pi \xi),
$$

where $a_{10}=\frac{12 \pi^{2} r^{2}\left(1-\nu^{2}\right) b^{2} A_{1}}{R h_{0}}$ and

$$
\begin{aligned}
f_{1}= & -\frac{a_{11}}{2 \pi^{4}\left(r^{2}+4\right)^{2}} \cos (2 \pi \xi)-\frac{e^{\pi r \xi}}{2 \pi^{4} r^{4}\left(r^{2}+4\right)^{2}\left(e^{\pi r}+1\right)^{2}}\left[e ^ { \pi r } \left(\pi r^{5} a_{12}+2 r^{4} a_{12}+\right.\right. \\
& \left.+2 \pi r^{3} a_{11}+8 \pi r^{3} a_{12}+8 r^{2} a_{11}+16 r^{2} a_{12}+16 \pi r a_{12}+8 \pi r a_{11}+16 a_{11}+32 a_{12}\right)+ \\
& \left.+2 r^{4} a_{12}+8 r^{2} a_{11}+16 r^{2} a_{12}+16 a_{11}+32 a_{12}\right]+\frac{1}{2 \pi^{4} r^{4}\left(r^{2}+4\right)^{2}}\left[16 a_{11}+32 a_{12}+\right. \\
& \left.+8 r^{2} a_{11}+2 r^{4} a_{12}+16 r^{2} a_{12}+r^{4} a_{11}\right]-\frac{e^{-\pi r \xi}}{2 \pi^{4} r^{4}\left(r^{2}+4\right)^{2}\left(e^{\pi r}+1\right)^{2}}\left[e ^ { 2 \pi r } \left(8 r^{2} a_{11}+\right.\right. \\
& \left.+2 r^{4} a_{12}+16 a_{11}+32 a_{12}+16 r^{2} a_{12}\right)-e^{\pi r}\left(2 \pi r^{3} a_{11}+\pi r^{5} a_{12}+8 \pi r a_{11}+16 \pi r a_{12}+\right. \\
& \left.\left.+8 \pi r^{3} a_{12}-8 r^{2} a_{11}-2 r^{4} a_{12}-16 a_{11}-32 a_{12}-16 r^{2} a_{12}\right)\right]+ \\
& +\frac{\left(r^{2} a_{12}+4 a_{12}+2 a_{11}\right) \xi e^{\pi r \xi}}{2 \pi^{3} r^{3}\left(r^{2}+4\right)\left(e^{\pi r}+1\right)}-\frac{\left(r^{2} a_{12}+4 a_{12}+2 a_{11}\right) e^{\pi r} \xi e^{-\pi r \xi}}{2 \pi^{3} r^{3}\left(r^{2}+4\right)\left(e^{\pi r}+1\right)},
\end{aligned}
$$


where:

$$
a_{11}=-a_{10}\left(r^{4}+4 r^{2}-\nu r^{2}+4\right), \quad a_{12}=\frac{2 a_{10}}{r^{2}+1} .
$$

Applying the Bubnov - Galerkin procedure to the equilibrium equation (35), arrive at:

$$
\begin{aligned}
& \int_{0}^{1} \int_{0}^{1}\left\{H^{3} \bar{w}^{(2.4)}+6 H^{2} \frac{d H}{d \xi} \bar{w}^{\prime \prime \prime}+\left[-6 r^{2} N^{2} H^{2} \frac{d H}{d \xi}\right] \bar{w}^{\prime}+\right. \\
& +\left[6 H\left(\frac{d H}{d \xi}\right)^{2}+3 H^{2} \frac{d^{2} H}{d \xi^{2}}-2 r^{2} N^{2} H^{3}+\frac{r^{2} b^{2} N_{x}^{0}}{D_{0}}\right] \bar{w}^{\prime \prime} \\
& \left.+\left[r^{4} N^{4} H^{3}-6 \nu r^{2} N^{2} H\left(\frac{d H}{d \xi}\right)^{2}-3 \nu r^{2} N^{2} H^{2} \frac{d^{2} H}{d \xi^{2}}\right] \bar{w}-\frac{b^{2}}{R h_{0}} \bar{f}^{\prime \prime}\right\} \delta \operatorname{sod}=\mathbf{0}
\end{aligned}
$$

where $\delta w=\sin (m \pi \xi) \sin (n \pi \eta)$.

Substituting (3.6) with $f_{i}$ into Eq.(3.13), making some algebraic manilation leads to an eigenvalue problem and the buckling load due to the thickness rintion can be determined.

\section{DETERMINATION OF THE BUCKLING LOAD}

In this paper, the expression for buckling loads are determined only when $\mathbf{r}=\boldsymbol{a} / \boldsymbol{b}=$ $1, \nu=0.3$ and $m=n=p=1$, using a single term displacement series obtained in (3.1), (3.2).

Equation (3.13) is given as the following:

$$
\left[-\frac{273}{100} \frac{\pi^{2} N^{c r}}{b^{2} E h_{0}^{3}}+\frac{\pi^{3}}{100 b^{4}}(100 \pi-660 \varepsilon)-\frac{13472}{10000} \frac{(9 \varepsilon-5 \pi)}{\pi^{3} h_{0}^{2} R^{2}}\right] \mathbf{A}=\mathbf{Q}
$$

where $A_{1}$ is the amplitude of deflection of the cylindrical panel $\left(A_{1} \neq \mathbf{C}\right.$ -

Consider the following normalization:

$$
\lambda=\frac{N^{c r}}{N_{0}^{c r}}
$$

where $\lambda$ is the non-dimensional buckling load factor due to the thict the buckling load of the cylindrical panel with constant thickness and $\boldsymbol{N}$ the buckling load of the cylindrical panel with variable thickness.

Substituting $\varepsilon=0$ into Eq. (4.1), the buckling load of the grind panel with constant thickness $\left(h=h_{0}\right)$ can be determined as:

$$
N_{0}^{c r}=0.0253 \frac{E h_{0} b^{2}}{R^{2}}+3.6153 \frac{E h_{0}^{\mathbf{3}}}{b^{2}} .
$$

When $\varepsilon \neq 0$, from Eq. (4.1), the buckling load of the cylindrical pand with variable thickness $h=h(x)$ is given: 


$$
N^{c r}=0.0253 \frac{E h_{0} b^{2}}{R^{2}}+3.6153 \frac{E h_{0}^{3}}{b^{2}}-\left(0.0145 \frac{E h_{0} b^{2}}{R^{2}}+7.5951 \frac{E h_{0}^{3}}{b^{2}}\right) \varepsilon .
$$

Substituting Eq.(4.3) and (4.4) into Eq. (4.2), the non-dimensional buckling load factor due to the thickness variation is obtained as follows :

$$
\lambda=1-\left(\frac{0.0145 b^{4}+7.5951 h_{0}^{2} R^{2}}{0.0253 b^{4}+3.6153 h_{0}^{2} R^{2}}\right) \varepsilon .
$$

\section{NUMERICAL ANALYSIS, COMPARISON AND DISCUSSION}

In Eq.(4.3), if $R \rightarrow \infty$ then the cylindrical panel will be the square plate, we obtain the same as the Timoshenko formula for a square plate with constant thickness in the form [1] $(r=a / b=1, \nu=0.3)$ :

$$
N_{0}^{c r}=\frac{\pi^{2} D}{b^{2}}\left(\frac{a}{b}+\frac{b}{a}\right)^{2}=\frac{\pi^{2} D}{b^{2}}(1+1)^{2}=\frac{4 \pi^{2} E h_{0}^{3}}{12 b^{2}\left(1-\nu^{2}\right)}=3.6153 \frac{E h_{0}^{3}}{b^{2}} .
$$

In this case, from Eq. (4.5), the non-dimensional buckling load factor due to the thickness variation of the square plate is given:

$$
\lambda=1-2.1 \varepsilon \text {. }
$$

The effect of thickness variation parameter $\varepsilon$ on the buckling load factor $\lambda$ is studied. The following figures are presented for cylindrical panel with $r=a / b=1$ and $\boldsymbol{\nu}=\mathbf{0 . 3}$.

Buckling of the perfect cylindrical panel with variable thickness: from Eq. (4.5), the relationship between $\lambda$ and $\varepsilon$ is shown in Fig. 3, the relationship between $\lambda$ and $R$ is shown in Fig. 4, the relationship between $\lambda$ and $\varepsilon, R$ is shown in Fig. 5.

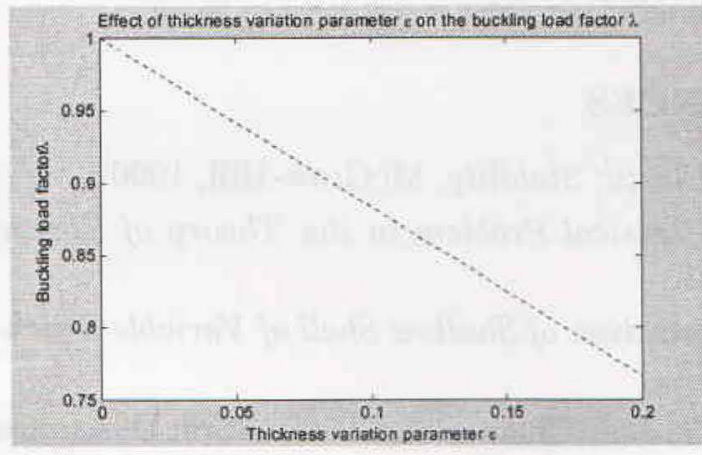

Fig. 3. Relation between $\lambda$ and $\varepsilon\left(\frac{R}{b}=2, \frac{b}{h_{0}}=30\right)$

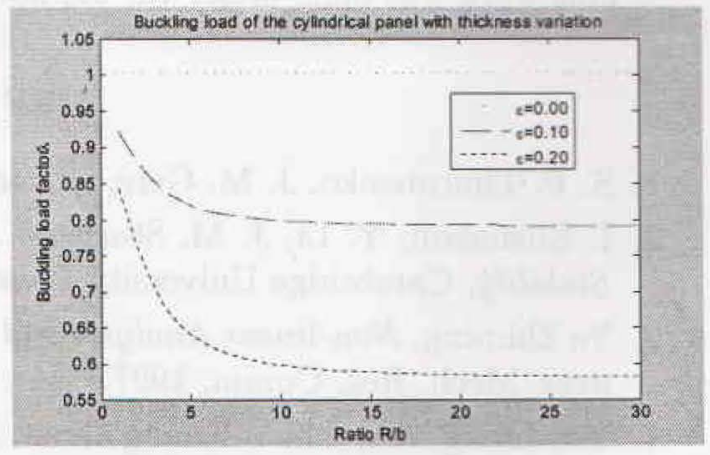

Fig. 4. Relation between $\lambda$ and $\frac{R}{b},\left(\frac{b}{h_{0}}=30\right)$ 


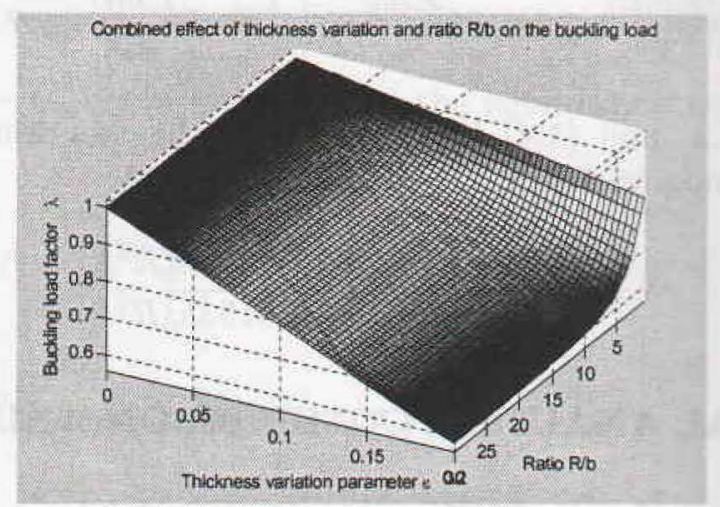

Fig. 5. Relation between $\lambda$ and $\varepsilon ; \frac{R}{b}\left(\frac{b}{h_{0}}=30\right)$

The results obtained show that the effect of thickness variation when $\varepsilon$ is positive. Even if the amplitude of the thickness variation is as small es $0.2,+1 \%$ chling load factor of cylindrical panel is reduced about $42 \%$ from its counterpart of the case with constant thickness, when $\frac{R}{b}=2$ and $\frac{b}{h_{0}}=30$ (Fig. 4 and Fig. 5).

\section{CONCLUSION}

In this paper, the coupled linearized governing stability equations for $\%$ indrical panel with variable thickness have been firstly introduced. Based on these equetions, a detailed study of the stability of the perfect cylindrical panel with thickness along the $x$-axes with sine functions has been presented. The formulae for the buckling load have been derived using the hybrid perturbation - Galerkin method.

From the obtained results, one can conclude that the variable thichess can cause a reduction of the load carrying capacity of cylindrical panel structures, and so this effect should be taken into account in the design of cylindrical panel structries.

This work was done under the support of Natural Science Council of Vietram.

\section{REFERENCES}

1. S. P. Timoshenko, J. M. Gere, Theory of Elastic Stability. McGraw-Hill, 1990.

2. I. Elishakoff, Y. Li, J. M. Starners, Non-Classical Problem in the Theory of Elastic Stability, Cambridge University Press, 2001.

3. Ye Zhiming, Non-linear Analysis and Optimization of Shallow Shell of Variable Thickness, Mech. Res. Comm, 1997.

4. Yen-Liang Yeh, Cha'o-Kuang Chen, Hsin-Yi Lai, Chaotic and Bifurcation Dynamics for a Simply Supported Rectangular Plate of Thermo-Mechanical Coupling in Large Deflection, Chao's, Solution and Fractals 13 (2002) 1493-1506.

5. A. F. Mateus, J. A. Wits, Post-Buckling of Corroded Steel Plates: A Comparative Analysis. 
6. Nguyen T. Hien Luong, Tran H. Tri, Influence of variable thickness on stability of rectangular plate under compression, Mechanics Research Communications 32 (2005) (2) $139-146$.

7. V. D. Cliusnhicov, Lectures on Stability of Deformable Solids, Publisher of Moscow University, 1986. (in Russian).

8. C. Y. Chia, Nonlinear Analysis of Plates, McGraw-Hill, N. Y., 1980.

9. D. T. Minh Tuong, Study of imperfect plate structures with a large deflection and variable thickness, Thesis of Master Dissertation in Civil Engineering, HCMC university of Technology, 2004.

Received October 28, 2005

Revised May 20, 2006

\section{PHÂN TÍCH ỔN DỊNH PANEL TRỤ CÓ CHIỀU DÀY THAY DỔI}

Bài báo phân tích ổn định của panel trụ có chiều dày thay đổi theo qui luật hình sin dựa trên lý thuyết panel trụ có độ võng nhỏ. Lực tới hạn của panel trụ tựa dơn quanh biên được xác định bằng phương pháp lai Nhiễu loạn- Galerkin. Ảnh hướng của chiều dày thay đổi tới lực tới hạn được khảo sát. 